Meta

Journal des traducteurs

Translators' Journal

\title{
La terminologie multilingue : une aide à la maîtrise des concepts
}

\section{Philippe Thoiron}

Volume 39, numéro 4, décembre 1994

Hommage à Bernard Quemada : termes et textes

URI : https://id.erudit.org/iderudit/004482ar

DOI : https://doi.org/10.7202/004482ar

Aller au sommaire du numéro

Éditeur(s)

Les Presses de l'Université de Montréal

ISSN

0026-0452 (imprimé)

1492-1421 (numérique)

Découvrir la revue

Citer cet article

Thoiron, P. (1994). La terminologie multilingue : une aide à la maîtrise des concepts. Meta, 39(4), 765-773. https://doi.org/10.7202/004482ar
Résumé de l'article

Le statut et le rôle de la traduction sont forcément différents selon qu'on envisage les sociétés unilingues ou les sociétés plurilingues. Dans une société unilingue la traduction substitue à un discours réputé inaccessible aux membres de la communauté un texte dans la langue vernaculaire. Dans une société multilingue, le même texte-source doit être traduit en plusieurs langues-cibles. On autorise ainsi, de facto , une approche multilingue des divers domaines de la connaissance. Un étudiant bilingue connaît la terminologie de son domaine d'étude dans deux langues, même si l'une d'elles a sa préférence. Il n'y a pas substitution d'une terminologie à une autre mais addition. La question soulevée ici concerne la valeur de cette addition. En particulier, on peut se demander si cette situation peut conduire à une meilleure maîtrise des concepts de la discipline à étudier. On se concentre ici sur les aspects formels et on suggère que la juxtaposition et l'addition d'informations extraites de la forme de termes homologues dans diverses langues sont utiles. Elles peuvent favoriser une compréhension plus fine de la structure des concepts. Enfin, grâce à l'approche comparative qu'elle induit, la terminologie multilingue peut être un vecteur non négligeable pour la prise de conscience chez les étudiants du rôle de la diversité culturelle dans les sciences et les techniques.
Tous droits réservés @ Les Presses de l'Université de Montréal, 1994
Ce document est protégé par la loi sur le droit d'auteur. L’utilisation des services d’Érudit (y compris la reproduction) est assujettie à sa politique d'utilisation que vous pouvez consulter en ligne.

https://apropos.erudit.org/fr/usagers/politique-dutilisation/ 


\title{
LA TERMINOLOGIE MULTILINGUE : UNE AIDE À LA MAÎTRISE DES CONCEPTS'
}

PHILIPPE THOIRON

Université Lumière Lyon-2, Lyon, France

\begin{abstract}
Résumé
Le statut et le rôle de la traduction sont forcément différents selon qu'on envisage les sociétés unilingues ou les sociétés plurilingues. Dans une société unilingue la traduction substitue à un discours réputé inaccessible aux membres de la communauté un texte dans la langue vernaculaire. Dans une société multilingue, le même texte-source doit être traduit en plusieurs langues-cibles. On autorise ainsi, de facto, une approche multilingue des divers domaines de la connaissance.

Un étudiant bilingue connâ̂t la terminologie de son domaine d'étude dans deux langues, même si l'une d'elles a sa préférence. Il n'y a pas substitution d'une terminologie à une autre mais addition. La question soulevée ici concerne la valeur de cette addition. En particulier, on peut se demander si cette situation peut conduire à une meilleure mâtrise des concepts de la discipline à étudier.

On se concentre ici sur les aspects formels et on suggère que la juxtaposition et l'addition d' informations extraites de la forme de termes homologues dans diverses langues sont utiles. Elles peuvent favoriser une compréhension plus fine de la structure des concepts. Enfin, grâce à l'approche comparative qu'elle induit, la terminologie multilingue peut être un vecteur non négligeable pour la prise de conscience chez les étudiants du rôle de la diversité culturelle dans les sciences et les techniques.
\end{abstract}

\begin{abstract}
The status and role of translation are necessarily different, according to whether we are thinking of multilingual or unilingual societies. In a unilingual society, translation is meant to replace the original text in a foreign language by another one written in the vernacular. In a multilingual society, the same original text has to be translated into several target languages, thus allowing de facto a multilingual approach to the various fields of knowledge.

When a bilingual student specializes in a field, he is familiar with its terminology in both languages, even though one language may have his preference. The essence of translation is then addition, not substitution. The question raised here deals with the value of such additions, particularly with respect to a better mastery of the concepts of the field of study.

We concentrate on the formal aspects and suggest that the addition of information extracted from the morphology of homologous terms in various languages can be useful. It can help the student to gain insight into conceptual structure and make him more aware of the part played by cultural diversity in the sciences and techniques.
\end{abstract}

Dans une société multilingue, la traduction a forcément un statut différent de celui dont elle jouit dans les sociétés unilingues. À côté de la traduction-substitution, qui remplace un discours réputé inaccessible aux membres de la communauté tout entière par un texte dans la langue vernaculaire, on peut envisager une traduction-addition, qui permet ou encourage la coexistence de textes traduits en plusieurs langues. On ne s'intéressera ici qu'au discours spécialisé, à l'exclusion de la langue commune, et encore à une partie seulement de ce discours, à savoir sa terminologie.

Meta, XXXIX, 4, 1994 
Un étudiant bilingue qui se spécialise dans un domaine lit les textes nécessaires à sa formation dans ses deux langues, même si l'une d'elles a sa préférence. Il connaît la terminologie de son domaine d'étude dans les deux langues. Il n'y a donc pas substitution d'une terminologie à une autre mais addition. La question que l'on peut poser concerne la valeur de cette addition, ou au moins de cette coexistence, de deux terminologies. En particulier, on peut se demander si cette situation peut conduire à une meilleure maîtrise des concepts de la discipline à étudier.

On se concentrera ici sur les aspects formels et on s'interrogera sur l'intérêt de la juxtaposition, de l'addition d'informations extraites de la forme des termes dans diverses langues.

\section{ÉCLAIRER LE SIGNIFIANT : UNE NÉCESSITÉ EN DIDACTIQUE ?}

Dans une culture donnée, avec une langue donnée et dans un domaine précisé, on considérera le concept comme unité d'un système (dit «conceptologie») fonctionnant au niveau cognitif. Le concept est désigné, dans le cadre idéal d'une relation biunivoque, par un terme fonctionnant, au niveau linguistique, au sein d'une terminologie.

Un concept peut être décrit comme étant constitué d'éléments plus petits (les traits conceptuels) ${ }^{2}$. En terminologie, l'accès à l'ensemble des traits conceptuels constitutifs d'un concept se fait par divers moyens: l'interrogation des spécialistes du domaine, et l'observation des comportements discursifs des termes, sachant que celle-ci est moins riche d'enseignements que pour les mots de la langue générale. En revanche, on peut souvent se servir de la morphologie des termes.

Une question fondamentale dans le cadre de la didactique d'une discipline est en effet celle de la transparence (on pourrait dire motivation) du signifiant. Un signifiant est d'autant plus transparent qu'un plus grand nombre des traits conceptuels du concept correspondant est intégré dans le processus de nomination. L'exemple du français LOUPE permettra d'illustrer ce point. Le signifiant ne dit rien du concept. En revanche, l'équivalent anglais magnifying glass («verre qui grossit») donne accès directement à deux traits conceptuels du concept LOUPE: la matière et la fonction.

Pourquoi le terme français est-il opaque? Un signifiant n'est pas opaque par hasard. Il se trouve que les procédés de nomination sont nombreux et divers. Dans ce cas on a eu affaire à une métaphore qu'on ne sait plus déchiffrer en français contemporain. Le terme vient du francique *luppa qui désignait une masse informe de liquide caillé. Au XIVe siècle et par métaphore on utilise loupe pour désigner une pierre précieuse d'une transparence imparfaite. Les propriétés optiques de ce type de pierre expliquent vraisemblablement le sens actuel. Mais il faut reconnaître que, si l'on ignore cette filiation étymologique, le signifiant de loupe est totalement opaque.

Un autre exemple bien connu fondé sur la démotivation d'une métaphore concerne la dénomination de la vitre arrière d'une automobile. Bien que l'objet n'ait plus aujourd'hui la forme ronde initiale qui motivait le diminutif formé sur lune, c'est le terme lunette qui est toujours employé. Il convient de relever cette «possibilité qu'offre la langue de conserver leur dénomination originelle à des objets différents fonctionnellement identiques» (Corbin 1992: 13) en sachant bien que cette faculté n'est qu'un corollaire de l'opacification de la motivation initiale.

La métaphore est un procédé important de dénomination dans le monde des sciences et des techniques. Ce procédé aboutit le plus souvent à la dénomination globale du concept et non pas à une vision analytique. Un autre exemple peut illustrer cela. On a appelé les verres convexes lentilles de verre (aujourd'hui lentilles simplement) en prenant le nom du légume ${ }^{3}$ mais on a donné aux verres concaves, utilisés pour la correction de la myopie, le nom de verres creux car il n'existe pas de légumes ayant cette forme. 
Toute la question ici consiste à savoir si lentille est plus intéressant que verres creux en décodage et du point de vue du contenu informationnel par rapport au concept. On a le sentiment que le signifiant verre creux renseigne davantage sur le concept associé que lentille.

La métonymie est un autre procédé fort utilisé pour la dénomination. Là aussi le signifiant peut être assez opaque lorsqu'il s'agit de métonymie codée (Voltaire pour fauteuil) alors que la métonymie structurelle (voile pour voilier) est plus transparente au décodage. L'exemple suivant illustre ce point. Dans le cadre de ses recherches la société IBM invente un disque dur dont les têtes de lecture, sur les deux faces, sont séparées du disque luimême par des coussins d'air. La capacité de ce disque dur est de 30 mégaoctets par face. Or, il existe dans la culture américaine une carabine de marque Winchester dite 30/30. Le premier 30 indique le calibre, le deuxième la charge de poudre noire exprimée en grains. Comme le disque avait 30 mégaoctets par face IBM l'a appelé disque Winchester. L'opacité de ce signifiant est totale, pour un Français par exemple. Aucun des traits constitutifs du concept n'a servi de base au processus de nomination ${ }^{4}$.

L'opacité du signifiant peut exister même si les traits conceptuels sont nommés à l'aide d'éléments de nomination5. C'est ce qui se produit lorsque les éléments constitutifs du terme ne sont pas, ou plus, perçus ou compris par les locuteurs. Le cas est fréquent en français avec ce qu'on a appelé les composés savants, termes formés sur des racines grecques ou latines. Quel Français moyen, n'ayant pas étudié le grec, comprendra que pirogue monoxyle désigne une embarcation creusée dans un seul tronc d'arbre? La racine xylon ne fait pas partie de son stock de morphèmes. L'Allemand moyen n'aura, lui, aucune peine à accéder au même concept puisque les morphèmes constitutifs de Einbaumboot sont connus de tout germanophone ${ }^{6}$. De la même manière, les équivalents arabes de certains termes français d'optique sont relativement transparents. On donne ci-dessous des gloses qui reproduisent, en français, le sens des termes trouvés dans divers dictionnaires scientifiques et techniques incluant de l'arabe (unilingues ou bilingues avec de l'arabe) ou dans des manuels arabes?.

$\begin{array}{ll}\text { français } & \text { glose littérale de l'arabe } \\ \text { dextrogyre } & \text { de droite en rotation } \\ \text { lévogyre } & \text { de gauche en rotation } \\ \text { interfrange } & \text { distance séparant deux franges } \\ \text { interfrange } & \text { distance entre deux franges } \\ \text { goniomètre } & \text { mesureur d'angles }\end{array}$

\section{MULTILINGUISME ET PLURALITÉ D’ÉCLAIRAGE CONCEPTUEL}

Même quand un signifiant est transparent, au sens où on l'a entendu ci-dessus, il est rare qu'il soit exhaustif. En d'autres termes, tous les traits constitutifs du concept ne sont pas nommés. Ceci n'a rien d'étonnant puisque la nomination ne doit pas être assimilée à la description, ou à la définition du concept. Quelques exemples permettent d'illustrer ce point.

Le français électrophone a pour équivalent anglais record-player. Le terme français, qui date de la fin du XIX $\mathrm{X}^{\mathrm{e}}$ siècle, ne fait pas mention du disque. Le terme anglais, défini dans le Dictionnaire d'Oxford comme «apparatus for reproducing sound from gramophone records» ne fait, lui, aucune allusion à l'électricité.

Dans le même domaine, le français disque est généralement traduit en anglais par record. Le Dictionnaire d'Oxford traite la polysémie de ce terme de la façon suivante :

1 - «trace made by recording instrument on disc or cylinder for subsequent reproduction by gramophone»

2 - «disc ... bearing such trace». 
On observera que le français met l'accent sur la forme de l'objet, l'anglais privilégie sa finalité.

Le français règle à calcul a pour équivalent anglais slide rule. Le concept, qui doit être vu comme une entité extra-linguistique, inclut un certain nombre de propriétés, ou de traits. Entre autres, ici,
1. |RÈGLE|
2. ICALCULER|
3. ICOULISSER / GLISSER I

Les traits conceptuels nommés peuvent alors être intégrés dans un modèle relationnel comparable à celui que Pottier établit pour POMPIER (1992a: 202)8. Or, toutes ces propriétés, qui sont nécessaires à la connaissance complète du concept, ne sont pas reprises dans le processus de nomination. En français, on a 1 +2 , en anglais $1+3$. Aucune de ces deux langues n'épuise toutes les possibilités. Il faudrait que le terme français fût *règle à calcul coulissante pour que cette condition fût remplie. Ce n'est évidemment pas le cas: un terme doit être maniable afin qu'il puisse être intégré dans un discours.

Si l'on sort du cadre strictement bilingue (anglais $\longrightarrow$ français, ou arabe $\longrightarrow$ français) utilisé jusqu'ici, on augmente les chances d'obtenir des informations nouvelles, par le biais de nouveaux éléments de nomination. Boisson (à paraître) a étudié les dénominations de la règle à calcul dans une quarantaine de langues, parmi lesquelles les langues majeures de la technique. On trouvera ci-dessous, à titre d'exemple, neuf de ces dénominations, correspondant à sept langues, ainsi que les gloses en français des éléments de nomination utilisés.

\begin{tabular}{|c|c|c|c|}
\hline anglais & $\begin{array}{l}\text { TERME } \\
\text { slide rule }\end{array}$ & $\begin{array}{l}\text { T DE NOMINATION } 1 \\
\text { «RÈGLE» }\end{array}$ & $\begin{array}{c}\text { ÉLÉMENT DE NOMINATION } 2 \\
\text { «COULISSER / GLISSER }\end{array}$ \\
\hline français $_{1}$ & règle à calculer & «RÈGLE» & «CALCULER» \\
\hline français $_{2}$ & règle logarithmique & «RÈGLE»» & «LOGARITHMIQUE» \\
\hline talien & regolo calcolatore & «RÈGLE» & «CALCULER» \\
\hline allemand & Rechenschieber & «COULISSEAU» & «CALCUL» \\
\hline USS & logarifmitcheskaja linejka & «RÈGLE» & «LOGARITHMIQUE» \\
\hline tchèque $_{1}$ & logaritmiké pravítko & «RÈGLE» & «LOGARITHMIQUE» \\
\hline tchèque $_{2}$ & pocítací pravítko & «RÈGLE» & «CALCULER» \\
\hline ébreu & sargel hichouv & «RÈGLE» & «CALCULER» \\
\hline
\end{tabular}

Ceci permet de reconstruire, pour chacune de ces langues, un embryon de concept. La réunion de ces ensembles embryonnaires de traits conceptuels 9 constituerait l'embryon de ce qu'on a appelé ailleurs «archi-concept (Thoiron et al. à paraître) et que l'on pourrait et que l'on pourrait représenter ainsi :

\{|RËGLEI, ICALCULERI, ICOULISSER / GLISSERI, |LOGARITHMIQUE|\}

L'étude exhaustive des dénominations de la règle à calcul (Boisson, à paraître) fait apparaître des éléments de nomination supplémentaires : l'un est totalement nouveau («servant à l'ingénieur» dans un terme arabe utilisé au Liban ${ }^{10}$ ), d'autres sont des variantes d'éléments de nomination déjà repérés («BÂTON», «BAGUETTE», «PLANCHETTE» qui renvoient tous à «RÈGLE»). On en est donc maintenant, pour l'archi-concept, à la représentation synthétique suivante. :

\{ IRÈGLEl, |CALCULERI, ICOULISSER/GLISSER|, |LOGARITHMIQUE|, |POUR INGÉNIEURS|\}

On observera que les éléments de nomination énumérés ici concernent des catégories conceptuelles fortement liées à la nature du référent (i.e. instrument). Il s'agit de 
1- sa morphologie (IRÈGLEl),

2- son mode de manipulation (ICOULISSER / GLISSERI),

3- son utilisateur-type (IPOUR INGÉNIEURSI),

4- sa fonction (|CALCULERI),

5- son principe théorique (ILOGARITHMIQUE)).

Si l'on admet que ces éléments de nomination sont articulés dans une proposition définitionnelle (le schéma définitionnel panlinguistique de Boisson, à paraître) on arrive à une approximation empirique de l'analyse de l'archi-concept de la règle à calcul:

(1) RÈGLE (2) COULISSANTE (3) DESTINÉE TYPIQUEMENT AUX INGÉNIEURS (4) POUR CALCULER (5) PAR LES LOGARITHMES

L'exemple de la règle à calcul est particulièrement intéressant dans la perspective où l'on se place ici puisqu'il s'agit d'un artefact à usage précis et restreint. L'étude des autres termes, même si elle n'a pas été aussi fouillée, donne des informations qui confirment l'intérêt de l'approche multilingue en terminologie. Il ressort en effet de tout cela que, selon la langue, on éclaire, par le biais de la nomination, tel(s) ou tel(s) élément(s) constitutif(s) du concept. En proposant, pour un concept donné, des équivalents en plusieurs langues, on enrichit la description des éléments du concept puisqu'on allonge la liste des éléments dénommés.

On peut parfois, par ce biais, corriger une interprétation fautive. Le terme français bilentille de Billet a pour équivalent anglais Billet split lens. Une étude du signifiant du terme français laisse supposer qu'on a deux lentilles, alors que la même étude portant sur l'anglais conduit à penser qu'il s'agit d'une lentille coupée en deux. La définition trouvée dans un ouvrage français (Mathieu, Kastler et Fleury 1985) est sans équivoque et montre bien qu'une stratégie de décodage à partir du signifiant anglais permettra une meilleure approche du concept que la même stratégie portant sur le français :

«lentille coupée suivant un diamètre et dont on peut écarter les moitiés L1 et L2 [...] dans leur plan normalement à ce diamètre. Ces deux moitiés donneront d'un point lumineux $\mathrm{A}$ situé sur l'axe de symétrie [...] des images réelles $\mathrm{A} 1$ et A2 dont la distance varie avec l'écartement de $\mathrm{L} 1$ et $\mathrm{L} 2$. On observe des interférences non-localisées dans la région commune aux deux faisceaux (en masquant la lumière directe).»

On peut rendre un signifiant plus clair, ou aider à son élucidation, en proposant un signifiant dans une autre langue. La recherche de la motivation, ou de la transparence, est un but à poursuivre dans le cas de la néologie. On peut d'ailleurs évaluer les créations terminologiques selon ce critère. Ainsi, la traduction en français de deux termes américains de télédétection aérospatiale a posé quelques problèmes relatés par Depecker (1991). Le terme ground range image (lit. «image à distance au sol") a été traduit par image dystopographique, tandis que slant range image (lit. «image à distance oblique») a reçu pour équivalent image médistancée. On observera que les préfixes dys- et mé- (qui sont de sens voisins, font partie du stock morphémique de tout Français raisonnablement cultivé) portent sur des racines différentes, ce qui accroît la motivation des signifiants de cette paire pour laquelle la différenciation des concepts doit être nette :

«Les termes américains, établis par juxtaposition d'éléments, sont peu explicites pour le nonspécialiste et intraduisibles en l'état. Les termes français, créés de manière concertée, sont en revanche très motivés et permettent de se représenter très rapidement et adéquatement les concepts en cause : cette qualité est d'un grand intérêt notamment pour la traduction vers d'autres langues et pour l'enseignement des techniques d'analyse d'images.

Il est possible de voir ainsi, dans ce cas de structuration terminologique, un exemple de la manière dont la langue fixe les idées et met en forme le savoir.» (Depecker 1991 : 233) 
Il apparaît, dans tous ces exemples, que le même concept est dénommé différemment, mais il existe des cas où le même référent est vu différemment selon les cultures. Ceci apparaît notamment dans les termes où l'on a recours à des métaphores. Les termes suivants, empruntés au domaine de l'optique, illustrent bien ceci. Le même phénomène est appelé distorsion en coussinet en français, et pincushion distorsion en anglais. Ce qui peut être glosé par distorsion en pelote d'épingles. Le terme arabe équivalent serait glosé par distorsion en croissant de lune. C'est à cause de ces différences, qui peuvent conduire à des divergences, que l'on évoque les dangers des créations terminologiques fondées sur la métaphore. Quemada dit indirectement la même chose quand il évoque la communauté restreinte (que constituent les groupes professionnels) et l'identité d'expérience permettant «à chacun de comprendre immédiatement le sens d'une métaphore spontanée en percevant les liens qui l'unissent à la réalité désignée» (Quemada 1978: 1166). Pour éviter les écueils possibles au moment du décodage des métaphores, mieux vaut, semble-t-il, combiner communauté culturelle et communauté professionnelle : l'un des deux ne suffit pas.

\section{Terminologie multilingue $v s$ enseignement multilingue}

La juxtaposition, l'addition d'informations extraites de la forme des termes dans diverses langues doivent être envisagées comme des aides à une meilleure maîtrise des concepts. On veillera à ne pas confondre l'approche multilingue d'une terminologie, telle qu'elle est présentée ici, avec un enseignement bilingue (ou multilingue) de la discipline, concept dont la mise en œuvre ne manque pas de poser des problèmes redoutables.

Des exemples très intéressants viennent de pays qui utilisent une langue étrangère, ou plusieurs, comme véhicule de connaissances se superposant ainsi à l'expression autochtone, orale ou écrite. On ne s'intéressera ici qu'à la situation des pays du Sud. Il apparaît que, pour les locuteurs de ces pays, un enseignement bilingue (ou multilingue) consiste souvent en «la dégradation des deux codes linguistiques qui conduit plus au 'semilinguisme' qu'au bilinguisme» (Moatassime 1992: 62).

Certains chercheurs du Maghreb 11 considèrent que le dualisme arabo-français provoque chez de nombreux locuteurs jeunes un tiraillement entre le savoir-dire d'une langue et le savoir-écrire de l'autre. Il n'est pas exclu que les interférences de deux ou plusieurs langues puissent affecter les capacités de raisonnement, surtout si ce sont des arguments d'autorité, fondés sur une prétendue logique, qui tiennent lieu de justification. Les mêmes voient dans le bilinguisme la cause de retard scolaire, de déficiences cognitives, de confusion mentale et de graves troubles linguistiques (bégaiement et aphasie), sociaux (difficultés de fixation professionnelle) et affectifs (difficultés relationnelles). On ne s'étonnera pas alors de voir des arabes francophones condamner eux-mêmes l'introduction simultanée de deux ou plusieurs langues. «À quelques rares exceptions près [...] le bilingue tunisien apparaît aussi médiocre dans l'une que dans l'autre langue [...] Hésitations, recherches, emploi de sabir franco-arabe, blocage total [...] Double culture, certes, mais se traduisant par un double mutisme.» (Garmadi 1972)

À cette vision pessimiste on peut opposer les arguments des adeptes du multilinguisme. Celui-ci favoriserait la flexibilité mentale et un développement cognitif favorable à une meilleure appréhension de l'univers. Les implications philosophiques concernant la meilleure compréhension des hommes et des cultures ne sont pas absentes. Plusieurs études, conduites en particulier dans les pays de tradition bilingue ou multilingue (Suisse, Belgique, Canada) montrent les avantages d'un bilinguisme développé précocément ${ }^{12}$. Toutefois, les auteurs de ces travaux, effectués dans les pays industrialisés ou auprès de populations culturellement favorisées, expriment une réserve certaine et une mise en garde contre un enthousiasme simpliste vis-à-vis du bilinguisme et de sa généralisation. Il 
semble bien que la mise en œuvre soit difficile lorsque l'une des langues en cause occupe une position nettement dominante (Cortès 1986).

Les facteurs politiques et sociologiques ne peuvent pas être négligés. Dans beaucoup de pays, et notamment au Sud, l'enseignement bilingue est perçu comme élitiste. «L'apprentissage des langues continue d'être ce qu'il a toujours été : le privilège d'une élite.» (Lietti 1989: 34). Ou bien il est perçu comme utopique dans des États où une scolarisation élémentaire unilingue ne peut pas être garantie à tous; «Seul un système scolaire très développé, soutenu financièrement et ayant un support socioculturel et familial solide peut se permettre [...] une formation précoce entièrement bilingue, et même trilingue ou quadrilingue.» (Moatassime 1992: 70). À titre d'exemple, on peut s'interroger, avec Moatassime, sur le cas d'un jeune maghrébin qui, outre sa langue maternelle, arabe dialectal ou berbère, devrait acquérir l'arabe classique et le français, sans parler de l'anglais, qui apparaît de plus en plus nécessaire aux études longues, voire l'espagnol, pour des raisons de voisinage. Ces exigences ne sont pas scientifiquement injustifiées, mais elles sont tenues pour parfaitement irréalistes par ceux qui ont la charge de l'éducation de l'ensemble de la population.

On doit donc constater que, même si l'on met de côté les arguments politiques relatifs au maintien de l'identité culturelle, c'est la nature des objectifs, perçus comme exorbitants, qui reste un obstacle formidable au développement du multilinguisme. Le repli sur soi et l'unilinguisme militant risquent d'être proposés comme le terme obligé de 1'alternative.

Il nous semble que des voies moyennes méritent d'être explorées. Celle qui a été proposée ici, parce qu'elle met en jeu, à des fins strictement comparatives et cumulatives, plusieurs langues, n'attribue à aucune un statut dominant. Parce que les exemples, tirés de langues variées, peuvent, et doivent, être glosés dans la langue d'enseignement, la méthode est neutre par rapport au choix de celle-ci. Il n'est pas interdit d'espérer que la mise en contact, fût-elle minime, avec les langues étrangères suscite chez l'élève ou l'étudiant des vocations ou des motivations favorables à un apprentissage linguistique plus élaboré.

Si les approches multilingues peuvent être débarrassées des connotations négatives qui les escortent encore chez certains, c'est d'un point de vue strictement scientifique que pourra être abordée, à la lumière des observations et des exemples développés ici, la question de l'utilité d'une terminologie multilingue raisonnée dans le cadre de la didactique d'une discipline.

Il conviendrait d'étudier de façon plus systématique dans quelle mesure ces présentations multilingues peuvent, en particulier dans des communautés multilingues, servir, en même temps qu'à l'enseignement des langues, de renfort à celui d'autres disciplines. La terminologie multilingue, grâce à l'approche comparative qu'elle induit, peut être alors un auxiliaire didactique supplémentaire et un vecteur non négligeable pour la prise de conscience chez les étudiants du rôle de la diversité culturelle dans les sciences et les techniques.

En présentant diverses dénominations d'un même concept, dans plusieurs langues, et en explicitant les signifiants, on apporte sur le concept un éclairage neuf. Il est évident que cette activité de type linguistique ne peut se substituer à l'acquisition des concepts dans le cadre de leur discipline. La véritable connaissance est d'ordre référentiel et non pas seulement linguistique. On sait bien que connaître les noms, ou les termes, ce n'est pas connaître les choses et les concepts. Mais se priver d'une réflexion sur les termes et sur leur forme, c'est aussi renoncer à enrichir sa propre connaissance d'un domaine. Refuser de regarder les dénominations des autres langues, c'est persister à penser que toute excursion terminologique est une perte de temps. On nous permettra sans doute d'être ici d'un autre avis. 


\section{Notes}

1. Le texte suivant reprend une partie des êléments d'une communication donnée à l'Université de Fès (Maroc) dans le cadre d'un colloque consacré aux relations entre didactique des sciences et multilinguisme, et organisé conjointement par la Faculté des Lettres de l'Université de Fès et le Centre de Recherches en Terminologie et Traduction de l'Université Lumière Lyon-2. Le lecteur comprendra mieux ainsi pourquoi de nombreux exemples sont empruntés à la langue arabe et à la situation linguistique au Maghreb.

2. La notation utilisée ci-après est la suivante :

- terme, en italiques minuscules,

- CONCEPT, en majuscules droites,

- ITRAIT CONCEPTUELI, en petites majuscules et encadré de deux I

- «ÉLÉMENT DE NOMINATION», en petites majuscules et encadré de guillemets doubles.

- ARCHI-CONCEPT, en italiques majuscules.

3. Cette métaphore serait née en Italie vers 1280 (Lelubre 1992).

4. On a continué à nommer disque Winchester des disques durs dont la capacité n'est plus de 30 Mo, ce qui «démotive» encore davantage le signe.

5. Le concept d'élément de nomination a été développé dans Thoiron et al. (à paraître) dont on donne ici un extrait.

«De même qu'un concept est divisible en traits conceptuels, on peut considérer qu'un terme est constitué d'éléments de nomination, eux-mêmes composés de un ou plusieurs morphèmes, de sorte qu'il existe une hiérarchie :

$$
\text { terme } \rightarrow \text { éléments de nomination } \rightarrow \text { morphèmes }
$$

Le terme luxation congénitale de la hanche est composé de trois éléments de nomination (luxation, congénitale et de la hanche). L'élément de nomination luxation est, à son tour, composé de deux morphèmes.» Pour une langue donnée, un élément de nomination renvoie donc à un trait conceptuel.

6. Dans son Esquisse de la langue finnoise, Sauvageot cite le cas de ophtalmologue et de son équivalent finnois «docteur des yeux». Selon lui le finnois serait plus «démocratique» que le français puisque les termes savants y sont plus facilement compréhensibles, et les «malapropisms», qui font le délice des Anglais ou des Français «cultivés», plus rares.

7. Ces exemples sont empruntés à Lelubre (1992).

8. Pottier propose «un modèle relationnel, duquel on pourra dériver les composés [...] dans différentes langues:

$\begin{array}{lllll}\text { [quelqu'un } & & \begin{array}{l}\text { lutter contre } \\ \text { éteindre }\end{array} & \text { feu } & \text { (avec) pompe] } \\ 1 & & 2 & 3 & 4 \\ 3-2-1 & \begin{array}{l}\text { allemand } \\ \text { anglais }\end{array} & \begin{array}{l}\text { Feuerwehrmann } \\ \text { firefighter }\end{array} & \\ 3-1 & \text { anglais } & \text { fireman } & \\ 4-1 & \text { français } & \text { pompier } & \\ & \text { espagnol } & \text { bombero } & \\ 2-1 & \text { français } & \text { éteigneur.» }\end{array}$

9. Il faut bien insister sur le fait qu'il s'agit d'embryons de concept et non de concepts complets et achevés. Il est entendu qu'un locuteur natif a une représentation conceptuelle beaucoup plus riche. On sait en effet que tous les traits conceptuels n'ont pas un élément de nomination correspondant.

10. Un seul terme dans les 41 langues étudiếes fait intervenir cet élément de nomination. On a choisi de l'incorporer parce qu'avec l'approche adoptée ici, il convient de ne rien rejeter. Les critères de fréquence d'emploi ne sont pas pertinents. Tout nouvel élément de nomination est a priori susceptible de jeter un éclairage neuf sur le concept. Les études sur l'histoire de la règle à calcul (Boisson, à paraître) montrent que cet objet a servi à des usagers aussi différents que des jaugeurs, des fonctionnaires d'octroi, des douaniers, des éleveurs de bestiaux, des contremaîtres, etc. L'élément de nomination trouvé en arabe libanais est le seul exemple évoquant la catégorie des «usagers». À ce titre il aurait vertu de prototype.

11. Voir liste de travaux dans Camilleri 1983.

12. Voir Hamers et Blanc 1983 ; Mackey 1982 ; Siguan et Mackey 1986 ; Lietti 1989.

\section{RÉFÉRENCES}

BOISSON, Claude P. (à paraître) : «Les dénominations de la règle à calcul», $\mathrm{ms.}$

CAMLLERI, Carmel (1983) : L'identité déchirée, numéro spécial de Peuples méditerranéens, juillet-septembre 1983 , pp. $147-152$.

CORBIN, Danielle (1992) : «Point de vue linguistique sur les mots construits», Le Courrier du CNRS : Sciences cognitives, $\mathrm{n}^{\circ} 79$, pp. 12-13. 
CORTÈS, Jacques (1986) : «Bilinguisme : tout reste à faire», Dialogues, juin 1986, pp. 18-21.

DEPECKER, Loïc (1991) : «Traitement d'une paire terminologique : ground range image ef slant range image», Meta, 36-1, pp. 231-233.

GARMADI, Salah (1972): «Les problèmes du bilinguisme en Tunisie», Abdel-Malek et al., Renaissance du monde arabe, Duculot, Gembloux, pp. 309-322.

HAMERS, Josiane F. et Michel BLANC (1983) : Bilinguité et bilinguisme, Bruxelles, Mardaga.

LELUBRE, Xavier (1992): La terminologie arabe contemporaine de l'optique: Faits, théories, évaluation, Thèse Nouveau Doctorat, Université Lumière Lyon-2.

LIETTI, Anna (1989): Pour l'éducation bilingue, Lausanne, Favre,

MACKEY, William F.(1982) : International Bibliography on Bilingualism, Québec, Presses de l'Université Laval.

MATHIEU, J. P., KASTLER, A. et P. FLEURY (1985) : Dictionnaire de physique, $1^{\text {re }}$ édition 1983, Paris, Masson/Eyrolles.

MOATASSIME, Ahmed (1992) : Arabisation et langue française au Maghreb, Paris, PUF.

POTTIER, B. (1992a): Théorie et analyse en linguistique, $2^{\mathrm{e}}$ éd., Paris, Hachette.

POTTIER, B. (1992b) : Sémantique générale, Paris, PUF.

QUEMADA, B. (1978): «Technique et langage. La formation des vocabulaires français des techniques», Histoire des techniques. Technique et civilisation. Techniques et sciences, Encyclopédie de la Pléiade, Paris, Gallimard, pp. 1146-1240.

SIGUAN, Miquel et William F. MACKEY (1986) : Éducation et bilinguisme, Genève, Bureau International de l'Éducation.

THOIRON, Philippe, ARNAUD, Pierre, BÉJOINT, Henri et Claude BOISSON (à paraître) : «Terminologies multilingues et 'archi-concept' : quelques aspects cognitifs de la recherche en terminologie.» 